\title{
PERSONAS CON DISCAPACIDAD MENTAL O PSICOSOCIAL EN SITUACIÓN DE CALLE
}

\author{
Jonathan Saguchi Chávez \\ Alma Alejandra Soberano Serrano \\ Universidad Autónoma de Baja California
}

\section{Resumen}

Este artículo forma parte de la investigación en proceso denominada "Violación al derecho humano a la salud en agravio de personas con discapacidad mental o psicosocial en situación de riesgo" para obtener el grado de magíster en derecho. Tiene como fin exponer la problemática que se vive en Ensenada, Baja California, México, respecto al abandono por parte de las autoridades sanitarias del Estado mexicano hacia las personas con discapacidad mental o psicosocial en situación de calle, con lo que se violenta su derecho al acceso a la salud.

Es tan lamentable como cierto que las personas que padecen discapacidades mentales y que se encuentran en situación de calle conforman uno de los grupos más desprotegidos de nuestra sociedad; por sus características, son vulnerables a todo su entorno y tienen dificultad para hacer valer sus derechos humanos fundamentales, por lo que están expuestos a una amplia gama de violaciones de los mismos.

Esta problemática debe ser atendida en observancia de los derechos humanos que protege la Constitución Política de los Estados Unidos Mexicanos, de los instrumentos internacionales de los que forma parte y de las legislaciones competentes en materia de salud mental del Estado mexicano, para motivar a las propias autoridades en el ámbito de sus competencias, pues parecen indiferentes ante la problemática existente.

Palabras clave: derechos humanos, derecho a la salud, discapacidad mental o psicosocial, situación de calle.

El autor: Jonathan Saguchi Chávez, becario Conacyt en el programa de Maestría en Ciencias Jurídicas, de la Universidad Autónoma de Baja California. Cargo y filiación. Correo electrónico: jsaguchi@uabc.edu.mx

Alma Alejandra Soberano Serrano, profesora investigadora adscrita a la Maestría en Ciencias Jurídicas, de la Universidad Autónoma de Baja California. Correo electrónico: alma.soberano@uabc.edu.mx

Recibido: 30 de octubre de 2018; evaluado: 9 de diciembre de 2018; aceptado: 18 de marzo de 2019. 


\title{
HOMELESS PEOPLE WITH MENTAL OR PSYCHOSOCIAL DISABILITIES
}

\author{
Jonathan Saguchi Chávez \\ Alma Alejandra Soberano Serrano \\ Universidad Autónoma de Baja California
}

\begin{abstract}
This article is part of an ongoing research called "Violation of the Human Right to Health against People with Mental or Psychosocial Disability in Situation of Risk" to obtain a Master in Law degree. It aims to expose existing problems in Ensenada, Baja California, Mexico, regarding the abandonment by Mexican state health authorities toward homeless people with mental or psychosocial disabilities, thus violating their human right to access health services.

It is as regrettable as it is true that homeless people who suffer from mental disabilites are one of the most unprotected groups in Mexico. Due to their characteristics, they are extremely vulnerable to their entire environment and they lack the capacity to assert their fundamental human rights, which is why they are exposed to a wide range of violations of these rights.

This problem must be addressed in observance of the human rights protected by the Political Constitution of the United States of Mexico, international treaties of which it is a party, and by competent national legislations in matter of mental health, in order to motivate Mexican authorities within the scope of their competences, since they seem indifferent in the face of these problems.
\end{abstract}

Keywords: human rights, right to health, mental or psychosocial disability, street situation.

About the authors: Jonathan Saguchi Chávez, Conacyt Fellow in the MA in Legal Sciences program,at the Universidad Autónoma de Baja California. Email: jsaguchi@uabc.edu.mx

Alma Alejandra Soberano Serrano, research professor in the MA in Legal Sciences program, at the Universidad Autónoma de Baja California. Email: alma.soberano@uabc.edu.mx

Received: October 30, 2018; reviewed: December 9, 2018; accepted: March 18, 2019. 


\section{Introducción}

En México es muy común ver personas con discapacidad mental o psicosocial en situación de calle que deambulan sin rumbo fijo, con grados notoriamente altos de desnutrición, que hurgan en contenedores de basura con el fin de encontrar un poco de alimento para saciar su hambre y que viven en condiciones inhumanas que degradan su dignidad ${ }^{1}$ al máximo. Ellas carecen de la capacidad intelectual para dimensionar el peligro latente que significa vivir en tales condiciones, en virtud de que están sistemáticamente expuestas a un sinfín de riesgos.

Con los años, esta situación se ha vuelto normal en las sociedades contemporáneas, por lo que pasa desapercibida. ¿Quién no ha visto a alguna persona con estas características en las calles de su ciudad o de cualquier otra? ¿Alguna vez nos hemos preguntado quién es la autoridad responsable de velar por su salud? ¿Estas personas tienen derechos humanos? Y, si los tienen, ¿por qué se permite que se violen sistemáticamente, en especial el de recibir una atención médica especializada?

Quienes padecen trastornos mentales severos, conocidos como personas con discapacidad mental o psicosocial (en adelante, PcD mental o psicosocial) que están en situación de calle conforman uno de los grupos vulnerables que, desde nuestro punto de vista, deben de formar parte de las prioridades del Estado mexicano y, por supuesto, de todos aquellos en los que este fenómeno tenga lugar. Es indiscutible que estos individuos, al no contar con familiares conocidos que velen por su salud y sus cuidados, se hallan a su suerte, pues también son abandonados por el Estado, aunque tenga la responsabilidad internacional y constitucional de salvaguardar su salud.

\section{Derecho humano a la salud desde un enfoque internacional}

Las personas que padecen de trastornos mentales severos se ubican dentro del grupo de PcD mental o psicosocial. Son diversos los instrumentos que se encuentran en el sistema internacional de los derechos humanos que tienen como propósito la

Para la Convención internacional sobre la eliminación de todas las formas de discriminación racial, la dignidad humana es el derecho que tiene cada ser humano a ser respetado y valorado como ser individual y social, con sus características y condiciones particulares, por el solo hecho de ser persona. 
protección de la salud ${ }^{2}$ de las personas, en particular, de las PcD. Así pues, cabe mencionar los más importantes de los que es parte el Estado mexicano.

En el sistema universal de los derechos humanos tenemos la Declaración universal de los derechos humanos (1948), que es el pilar del sistema internacional de los derechos fundamentales. "A partir de ella se erige la premisa fundamental de la universalidad, indivisibilidad e interdependencia de los derechos civiles y políticos, y los derechos económicos, sociales y culturales". ${ }^{3}$ En su Artículo 25 tutela:

Toda persona tiene derecho a un nivel de vida adecuado que le asegure, así como a su familia, la salud y el bienestar, y en especial la alimentación, el vestido, la vivienda, la asistencia médica y los servicios sociales necesarios; tiene asimismo derecho a los seguros en caso de desempleo, enfermedad, invalidez, viudez, vejez u otros casos de pérdida de sus medios de subsistencia por circunstancias independientes de su voluntad. ${ }^{4}$

Dentro de la Carta internacional de los derechos humanos, el Pacto internacional de derechos económicos, sociales y culturales, adoptado y abierto a firma, ratificación y adhesión por la Asamblea General en su Resolución 2200 A (XXI), del 16 de diciembre de 1966 y que entró en vigor el 3 de enero de 1976, establece:

1) Los Estados Partes en el presente Pacto reconocen el derecho de toda persona al disfrute del más alto nivel posible de salud física y mental.

2) Entre las medidas que deberán adoptar los Estados Partes en el Pacto a fin de asegurar la plena efectividad de este derecho, figurarán las necesarias para: [...].

d) La creación de condiciones que aseguren a todos asistencia médica y servicios médicos en caso de enfermedad. ${ }^{5}$

2 La Organización Mundial de la Salud define la salud como un estado de completo bienestar físico, mental y social y no solo como la ausencia de afecciones o enfermedades.

3 Rocío Barahona-Riera, "Derechos económicos, sociales y culturales: exigibilidad —niveles mínimos de cumplimiento- e indicadores" (ponencia presentada en la Reunión de expertos sobre población, desigualdades y derechos humanos, Santiago de Chile, 26-27 octubre, 2006), 9.

4 Organización de Naciones Unidas [ONU], Declaración universal de los derechos humanos (Nueva York, 10 de diciembre de 1948), art. 25.

5 Organización de Naciones Unidas [ONU], Pacto internacional de derechos económicos, sociales y culturales (Nueva York, 16 de diciembre de 1966), art. 12. 
Ello, claro está, sin pasar por alto el sistema interamericano de derechos humanos, en especial la Convención americana sobre los derechos humanos, también conocida como Pacto de San José.

Ahora bien, en materia de protección de derechos humanos de las PcD, el que consideramos más importante es la Convención sobre los derechos de las personas con discapacidad y su Protocolo facultativo, ${ }^{6}$ aprobados por la Organización de Naciones Unidas el 13 de diciembre de 2006. De ella se destaca la alta participación de los Estados miembros, ya que en la historia de la ONU no existían precedentes de tan alta aceptación y tantos signatarios.

Esta Convención señala un cambio paradigmático de las actitudes y los enfoques respecto a las personas con discapacidad. Se concibió como un instrumento de derechos humanos con una dimensión explícita de desarrollo social, en la cual se adopta una amplia clasificación de las PcD y se reafirma que todas las personas con todos los tipos de discapacidad deben poder gozar de todos los derechos humanos y las libertades fundamentales. ${ }^{7}$ Se aclara y precisa cómo se aplican a las PcD todas las categorías de derechos y se indican las esferas en las que es necesario introducir adaptaciones para que las $\mathrm{PcD}$ puedan ejercer en forma efectiva sus derechos, aquellas en las que se han vulnerado y en las que debe reforzarse la protección. ${ }^{8}$

En relación con la protección de la salud de las PcD, el Artículo 25 expresa que los Estados parte adoptarán las medidas pertinentes para asegurar el acceso de las PcD a los servicios de salud que tengan en cuenta las cuestiones de género, incluida la rehabilitación. ${ }^{9}$ Esta Convención, como muchas otras, viene acompañada por un protocolo facultativo, es decir, precisiones sobre todo procesales o de actuación acerca de lo instituido en ella. Es "facultativo" en el sentido de que los Estados no están obligados a ser parte en el Protocolo, aun cuando lo sean en el tratado original. ${ }^{10}$

La Convención sobre los derechos de las personas con discapacidad fue aprobada por la Asamblea General de la Organización de Naciones Unidas en su Resolución 61/106, durante su sexagésimo primer período de sesiones en Nueva York, el 13 de diciembre de 2006.

7 Centro de información de la Organización de Naciones Unidas [ONU], "Día internacional de las personas con discapacidad", http://cinu.mx/eventos/observancia/dia-internacional-de-las-perso-4/ (acceso octubre 26, 2018).

8 Centro de información de la ONU, "Día internacional de las personas con discapacidad".

9 Carlos-Eloy Ferreirós Marcos, Salud mental y derechos humanos: la cuestión del tratamiento ambulatorio involuntario (Madrid: Comité español de representantes de personas con discapacidad, 2007), 254.

10 Organización de las Naciones Unidas [ONU], De la exclusión a la igualdad. Hacia el pleno ejercicio de los derechos de las personas con discapacidad (Ginebra: SRO-Kundig, 2007), 47. 
El Protocolo facultativo de la Convención sobre los derechos de las personas con discapacidad sostiene:

Todo Estado Parte en el presente Protocolo ("Estado Parte") reconoce la competencia del Comité sobre los Derechos de las Personas con Discapacidad ("el Comité") para recibir y considerar las comunicaciones presentadas por personas o grupos de personas sujetos a su jurisdicción que aleguen ser víctimas de una violación por ese Estado Parte de cualquiera de las disposiciones de la Convención, o en nombre de esas personas o grupos de personas. ${ }^{11}$

El artículo citado tutela procedimientos que están diseñados para una mejor eficacia en cuanto a la observancia de la Convención y define dos procedimientos para este fin: uno de comunicaciones personales y uno de investigación.

El procedimiento de comunicaciones personales consiste en la facultad que les otorga el Protocolo facultativo a quienes se hayan visto violentados en sus derechos humanos por la inobservancia de la Convención para presentar sus denuncias ante el Comité y este, que ya cuenta con la aceptación de competencia por parte del Estado que ratificó el Protocolo, activará el procedimiento de validación de los elementos de admisibilidad, fundamentación y motivación que dio cabida a tal denuncia; de ser procedente, dará inicio a la investigación.

Una vez superada la parte de admisibilidad, si así lo considera, el Comité invitará al Estado denunciado a que colabore en el esclarecimiento de la denuncia interpuesta en su contra y le enviará copia de la denuncia para que manifieste lo que a su derecho convenga. ${ }^{12}$ Examinadas las manifestaciones del Estado denunciado — que regularmente tratan de desvirtuar la denuncia—, el Comité designará, si así lo considera necesario, a uno o más miembros para que lleven a cabo la investigación, quienes deberán redactar un informe de carácter urgente sobre las primeras aproximaciones resultantes. Esta indagación es confidencial y se ha de llevar a cabo con la plena colaboración del Estado. ${ }^{13}$

11 Organización de las Naciones Unidas [ONU], Protocolo facultativo de la Convención sobre los derechos de las personas con discapacidad (Nueva York, 13 de diciembre de 2006), art. 1.

12 Esta parte del proceso presenta mucha similitud a lo que, en materia de amparo, en el Estado mexicano conocemos como "informe justificado de la autoridad responsable".

13 ONU, De la exclusión a la igualdad, 48. 
La mayoría de tratados internacionales sobre derechos humanos admiten procedimientos facultativos de comunicaciones y otros tantos admiten procedimientos de investigación. No obstante, a la fecha no existe un instrumento jurídico internacional destinado a las PcD mental o psicosocial. Sin carácter vinculante, la Asamblea General de la Organización de Naciones Unidas adoptó los Principios para la protección de los enfermos mentales y la mejora de la atención de la salud mental ${ }^{14}$ mediante Resolución 46/119, del 17 de diciembre de 1991, que constituye un marco mínimo orientador. ${ }^{15}$

\section{Informe inicial del Estado mexicano sobre el cumplimiento de la Convención sobre los derechos de las personas con discapacidad y observaciones del Comité}

En cumplimiento del Artículo 35 de la CSDPCD, México presentó en abril de 2011 el primer informe exhaustivo sobre el cumplimento de dicha Convención ante el Alto Comisionado de la ONU, en el cual se evidencian las deficiencias tanto legislativas como organizacionales de las dependencias en materia de salud. México reconoce que:

La experiencia del país ha sido distinta dependiendo del tipo de limitación que presenta la población. Mientras que se registran mayores avances respecto a la aplicación de políticas relacionadas con la discapacidad física y sensorial, la atención a la discapacidad intelectual desde una perspectiva de derechos humanos presenta un mayor rezago. ${ }^{16}$

Refiere el informe que es tal el rezago, por no decir las violaciones a los derechos humanos de las personas con discapacidad mental o psicosocial, que ni siquiera hay una aproximación de la cantidad de seres humanos que lo padecen. ${ }^{17}$ Así lo expresa el informe:

\footnotetext{
Comisión Interamericana de Derechos Humanos, Principios para la protección de los enfermos mentales y el mejoramiento de la atención de la salud mental (Washington, 17 de diciembre de 1991), art. 1.

15 Ferreirós Marcos, Salud mental y derechos humanos, 254.

16 Secretaría de Relaciones Exteriores, Informe inicial de México sobre el cumplimiento de la Convención sobre los derechos de las personas con discapacidad (Ciudad de México: Secretaría de Relaciones Exteriores, Dirección general de derechos humanos y democracia, 2011), 11.

17 Se argumenta en el informe que en México se han hecho diversos esfuerzos para medir la prevalencia de la discapacidad desde hace varias décadas. Sin embargo, aún se desconoce la verdadera dimensión y el comportamiento histórico de este indicador, ya que se han utilizado diferentes conceptos, clasificaciones
} 
El análisis de la información disponible también sugiere que un porcentaje importante de la población con discapacidad intelectual es víctima de discriminación y a menudo se encuentra al margen del ejercicio de sus derechos humanos. Como reconoce el PRONADDIS, en la mayoría de los casos este grupo de la población tiene limitaciones para acceder, en igualdad de condiciones, a la educación, al empleo, a la protección social, a la salud, a la cultura, a los medios de transporte, a la información, a la vida política, así como a otros derechos básicos como formar una familia, disfrutar de la sexualidad, ejercer el derecho al voto o disfrutar de la vida social. ${ }^{18}$

El 3 de octubre de 2014, el Comité sobre los derechos de las personas con discapacidad, que es responsable de evaluar la implementación de la Convención en los países miembros, en ejercicio de sus facultades examinó el informe enviado por México. De su análisis sobresalen las siguientes observaciones y recomendaciones: ${ }^{19}$

Principales ámbitos de preocupación y recomendaciones

A. Principios y obligaciones generales (artículos 1 a 4)

5. Al Comité le preocupa que la legislación civil de algunas entidades federativas todavía contenga expresiones peyorativas para referirse a las personas con discapacidad.

6. El Comité recomienda al Estado parte que redoble sus esfuerzos en el ámbito del proceso de armonización legislativa con la Convención a fin de que todos los estados federales eliminen terminología peyorativa de los derechos de las personas con discapacidad.

7. Al Comité le preocupa que las organizaciones de personas con discapacidad tengan limitada su participación en la implementación y seguimiento de la Convención.

y fuentes de información con el paso del tiempo, que no han servido para identificar con exactitud la prevalencia de este fenómeno.

18 Secretaría de Relaciones Exteriores, Informe inicial de México, 15.

19 El Comité examinó el Informe inicial de México (CRPD/C/MEX/1) en sus sesiones $145^{\mathrm{a}}$ y $146^{\mathrm{a}}$ —celebradas los días 16 y 17 de septiembre de 2014 - y aprobó en su $167^{\text {a }}$ sesión —celebrada el 30 de septiembre de 2014 - 
8. El Comité recomienda al Estado parte que establezca mecanismos regulares para convocar consultas con las organizaciones de personas con discapacidad, asegurando que sus opiniones reciban la consideración adecuada.

Derechos específicos (artículos 5 a 30)

Igualdad y no discriminación (artículo 5)

9. Al Comité le preocupa la discriminación contra las personas con discapacidad en el Estado parte, que se ve agravada por la concurrencia de otros factores de exclusión social, como la edad, el género, la pertenencia étnica y la ruralidad. Asimismo, le preocupa que en algunos estados se encuentre pendiente la adopción de leyes que prohíban la discriminación por motivos de discapacidad y que reconozcan la denegación de ajustes razonables como forma de discriminación basada en la discapacidad.

10. El Comité recomienda al Estado parte que establezca líneas presupuestarias específicas para cumplir sus objetivos en materia de igualdad, así como acciones específicas para combatir casos de discriminación interseccional, basadas en la discapacidad, la edad, el género, la pertenencia a pueblos indígenas y la ruralidad, entre otros factores de exclusión. Del mismo modo, lo alienta a aumentar sus esfuerzos, desarrollando estrategias de difusión, toma de conciencia y diálogo con las autoridades locales, a fin de que todos los estados expidan legislación prohibiendo la discriminación basada en la discapacidad y reconozcan la denegación de ajustes razonables como forma de discriminación.

11. El Comité se encuentra preocupado por el reducido número de quejas y de pronunciamientos sobre casos de discriminación por motivos de discapacidad, por la falta de reglamentación de la Ley Federal para Prevenir y Eliminar la Discriminación y por la poca información sobre su difusión en formatos accesibles, incluyendo lenguas indígenas.

12. El Comité recomienda al Estado parte asignar recursos para que la Ley Federal para Prevenir y Eliminar la Discriminación sea traducida a todas las lenguas indígenas en formatos accesibles incluidos el braille, la lengua de señas, la lectura fácil y los formatos electrónicos. El Comité alienta al Estado parte a realizar campañas contra la discriminación de personas con discapacidad 
dirigidas a la profesión legal, incluyendo los funcionarios del poder judicial y los abogados. ${ }^{20}$

En las observaciones emitidas por el Comité no se aprecia pronunciamiento alguno sobre las PcD mental o psicosocial en situación de calle. Por supuesto, el informe presentado por el Estado mexicano tampoco hace referencia a este grupo sumamente vulnerable.

El 22 de febrero de 2018, México presentó combinados el segundo y el tercer informe ante el Comité. En ellos hace una serie de descripciones de la forma como ha implementado estrategias tanto legislativas como institucionales para el cumplimento de la CSDPCD. No obstante, de la lectura del documento completo no se desprende alguna estrategia concreta para combatir la problemática de las PcD mental o psicosocial en situación de calle.

\section{El derecho humano a la salud en la legislación mexicana}

El cambio de paradigma constitucional que surgió a partir de la importante reforma de la Constitución Política de los Estados Unidos Mexicanos (CPEUM) del 10 de junio de 2011 trajo cambios muy significativos en la vida jurídica del país, sobre todo en materia de derechos humanos.

En primer lugar, mediante la reforma del primer párrafo del Artículo l constitucional, se da por terminada la vieja y reiterada discusión sobre las garantías individuales, ya que pasamos de tener un Título Primero, de las garantías individuales otorgadas, a un Título Primero, de los derechos humanos y sus garantías, con lo que se elevaron a rango constitucional los derechos humanos. Este no es un simple cambio terminológico que solo haría que ese conjunto de los derechos se denominara de manera distinta. Su implicación es más profunda, pues se trata de un cambio radical en el modo como deberán ser entendidos, tratados e interpretados; es la adopción del entendimiento más amplio y universalmente aceptado de los derechos: por el simple hecho de ser humano, los tiene cualquier persona. ${ }^{21}$

20 Organización de las Naciones Unidas [ONU], Observaciones finales sobre el Informe inicial de México (Nueva York: Comité sobre los derechos de las personas con discapacidad, 2014), 2-3.

21 Karlos Castilla Juárez, "Un nuevo panorama constitucional para el derecho internacional de los derechos humanos en México", Estudios constitucionales 9, núm. 2 (junio-diciembre 2011): 127, https://scielo.conicyt. cl/pdf/estconst/v9n2/art04.pdf (acceso octubre 26, 2018). 
Sin duda, la mencionada reforma ha abonado un cambio positivo en el paradigma de los derechos humanos en México, pues no es exclusiva del Artículo 1, sino que armoniza la CPEUM con los estándares internacionales en la materia, al establecer un bloque de constitucionalidad que se enriquece con una amplia legislación que da las herramientas jurídicas —o al menos eso se pretende— para que los operadores del derecho lleven a cabo el control de constitucionalidad, es decir, "la sistematización jurídica de todas las normas materialmente constitucionales (Constitución, tratados internacionales, pactos, protocolos y legislación ordinaria)". 22

El bloque de constitucionalidad es una regla de reconocimiento de la norma constitucional, cuyo criterio de pertenencia no se limita a los contenidos normativos instituidos en un solo texto constitucional, sino que lo extiende a otras normas que comparten determinadas notas materiales o sustantivas. ${ }^{23}$ De esta manera, el derecho internacional de los derechos humanos en México cuenta desde el año 2011 con un nuevo panorama constitucional, pues ha pasado de ser un elemento integrante de la Ley Suprema de la Unión que poco se usaba a ser, por mandato del propio texto constitucional, fuente normativa de derechos, elemento integrante del bloque de constitucionalidad y, con ello, de la Constitución lato sensu. ${ }^{24}$

A grandes rasgos, esto es lo que ha acontecido en la reforma constitucional en cuanto al cambio de paradigma de los derechos humanos en nuestro país, al armonizar la legislación nacional al sistema internacional de los derechos humanos. La CPEUM instituye: "En los Estados Unidos Mexicanos todas las personas gozarán de los derechos humanos reconocidos en esta Constitución y en los tratados internacionales de los que el Estado Mexicano sea parte, así como de las garantías para su protección $[\ldots] " .{ }^{25}$

Se entiende que todo gobernado en territorio mexicano goza de los derechos humanos reconocidos en nuestra CPEUM y en los tratados internaciones de los que México es parte. Constitucionalmente, todo titular de derechos humanos debe ser tratado como igual dentro de sus propias circunstancias, pues: "Una sociedad

22 Eduardo Ferrer Mac-Gregor, "El control difuso de convencionalidad en el Estado constitucional" en Formación y perspectivas del Estado en México, ed. Héctor Fix-Zamudio y Diego Valadés (Ciudad de México: Universidad Nacional Autónoma de México, 2010), 172.

23 Castilla Juárez, "Un nuevo panorama constitucional", 147.

24 Castilla Juárez, "Un nuevo panorama constitucional", 148.

25 Estados Unidos Mexicanos, Constitución Política (Ciudad de México: Sista, 2017 [1917]), art. 1. 
igualitaria no significa que no existan diferencias entre las personas sino que se realice un enfoque positivo que permita acomodar tales diferencias en su seno". ${ }^{26}$

En el segundo y tercer párrafo del artículo en mención aparecen los principios de interpretación conforme, hermenéutico - también conocido como principio pro persona - de universalidad, de interdependencia, de indivisibilidad y de progresividad de los derechos humanos, definidos y desarrollados ampliamente por diversos instrumentos internacionales.

Luego, todas las PcD están protegidas y gozan de los derechos humanos tanto en materia constitucional como en materia convencional.

En atención al Artículo 4 del mismo cuerpo normativo supremo, reformado en 1983 por las exigencias internacionales en salud, se adicionó un tercer párrafo:

Toda persona tiene derecho a la protección de la salud. La Ley definirá las bases y modalidades para el acceso a los servicios de salud y establecerá la concurrencia de la Federación y las entidades federativas en materia de salubridad general, conforme a lo que dispone la fracción XVI del artículo 73 de esta Constitución [...]. ${ }^{27}$

Con esto nuestra legislación se alinea con los criterios establecidos por la Organización Mundial de la Salud (OMS) y por la Organización Panamericana de la Salud (OPS). Al adoptar los criterios establecidos por la OMS y la OPS, y conforme a lo establecido en el Artículo 133 constitucional, en el que se indica la obligatoriedad de considerar los tratados internacionales como leyes locales, se reafirma la necesidad de considerar el derecho a la salud como bien supremo y, por ende, como derecho fundamental. ${ }^{28}$

No obstante, es importante precisar que la fracción tercera del Artículo 4 que acabamos de analizar es de carácter programático, en virtud de que dispone una directriz dirigida a un órgano dependiente de un poder constituido — la Secretaría de Salud del Estado—, que se regirá por la Ley general de salud, reglamentaria del artículo en mención.

26 Ferreirós Marcos, La cuestión del tratamiento ambulatorio involuntario, 250.

27 Estados Unidos Mexicanos, Presidencia de la República, Decreto por el que se reforma y adicionan diversas disposiciones de la Constitución Politica de los Estados Unidos mexicanos (Ciudad de México: Diario Oficial de la Federación núm. xx, 20 de junio de 1983), art. 4.

28 Javier Saldaña, Derechos del enfermo mental (Ciudad de México: Instituto Politécnico Nacional, 2000), 22. 
El derecho humano a la salud —perteneciente a los derechos de segunda generación, conocidos como derechos económicos, sociales y culturales (DESC) — es de orden declarativo y prestacional, es decir, impone al Estado una obligación positiva de hacer — salvaguardar la salud de todos sus gobernados—; en este sentido, la norma constitucional transfiere la potestad de regular y normar los procedimientos y mecanismos para su protección a una ley secundaria.

La ley reglamentaria del Artículo 4, párrafo 3, es la Ley general de Salud, ${ }^{29}$ que está reglamentada por la Ley de salud pública perteneciente a cada entidad federativa que, en el caso que nos ocupa, es la Ley de salud pública para el estado de Baja California (LSPEBC), cuyo Artículo 11 dispone que corresponde al Ejecutivo del estado, por conducto de la Secretaría de Salud del estado, garantizar la salud ${ }^{30}$ de PcD mental o psicosocial; más aún, esta ley instituye que se le deberá dar prioridad a las personas que se encuentren en situaciones como la aludida en este artículo.

\section{La problemática en México}

El 22 de febrero de 2018, México entregó al Comité de la CSDPCD el segundo informe ${ }^{31}$ sobre la aplicación y observancia de la Convención sobre los derechos de las personas con discapacidad en el país. Hoy en día aún no hay recomendaciones al respecto y se desconoce si se presentaron informes sombra o alternativos. ${ }^{32}$ En el segundo y el tercer informes se incluyen las siguientes estadísticas:

El Censo de Población y Vivienda 2010 concluyó que el 5,1 \% de la población en México, equivalente a 5,7 millones de personas, eran PcD. La ENADID-2014, incluye en su instrumento de hogar el set corto de preguntas del GW y dos dominios más, y refiere que, de casi 120 millones de habitantes, 6 \% reporta discapacidad, equivalente a 7,1 millones de PcD.

29 La presente ley reglamenta el derecho a la protección de la salud que tiene toda persona en los términos del Artículo 4 de la Constitución Política de los Estados Unidos Mexicanos. Establece las bases y modalidades para el acceso a los servicios de salud y la concurrencia de la Federación y las entidades federativas en materia de salubridad general. Es de aplicación en toda la República y sus disposiciones son de orden público e interés social. Estados Unidos de México, Cámara de Diputados, Ley general de salud (Ciudad de México: Diario Oficial de la Federación, 7 de febrero de 1984), art. 4.

30 Estados Unidos de México, Congreso del estado, Ley de salud pública para el estado de Baja California (Mexicali: Periódico Oficial núm. 49, 9 de noviembre de 2001), art. 139.

31 El primer informe fue presentado en 2014, ante el Comité de la Organización de las Naciones Unidas.

32 Estos documentos pueden ser elaborados y presentados indistintamente por organizaciones no gubernamentales (ONG) o cualquier colectivo o no colectivo en defensa de los derechos humanos de personas con discapacidad. Su objetivo es informar la realidad sobre el cumplimento de la CSDPCD, sin maquillaje y sin cifras fantasmas, es decir, revelan las falacias que los Estados pudieran plasmar en los informes. 
La discapacidad tiene mayor presencia femenina en localidades urbanas $(54,7 \%$ mujeres y $45,3 \%$ hombres); y menor en localidades rurales (49,6\% mujeres y $50,4 \%$ hombres).

En 2014, del total de PcD, 64,1 \% presentó limitación para usar sus piernas y $58,4 \%$ visual, $33,5 \%$ refirió auditiva y $19,6 \%$ por dificultad mental o emocional. La mayoría de las discapacidades tuvieron su origen en alguna enfermedad (41,3\%),33,1 \% por edad avanzada, $10,7 \%$ por nacimiento, $8,8 \%$ por accidente, $0,6 \%$ por algún hecho violento y $5,5 \%$ reportan otra causa.

La prevalencia de discapacidad entre la población de 3 años y más que habla lengua indígena fue del $7,1 \%$, ligeramente más alta que la media nacional. Por sexo, el porcentaje fue similar, $7,1 \%$ en hombres y $7 \%$ en mujeres.

$83,3 \%$ de PcD estaban afiliadas a un servicio de salud frente al 79,8 \% de personas sin discapacidad. El porcentaje de mujeres fue 3,7 \% mayor que el de los hombres. El 52,7 \% de PcD estaba afiliada a instituciones de seguridad social y 50,5\% están cubiertas por los programas sociales. El 46,5 \% de PcD, de 3 a 29 años, asistía a la escuela contra 60,6 \% de población sin discapacidad, reportando los porcentajes más altos de asistencia escolar entre los 6 y los 14 años (nivel básico); posterior a estas edades, se presenta una reducción importante de asistencia escolar a partir de los 15 años.

La tasa de participación económica de PcD fue de 39,1\%. Una de cada cuatro $\mathrm{McD}$ participaban en actividades económicas y en el caso de los hombres, la proporción es de uno por cada dos. La tasa de participación económica varía según el tipo de discapacidad, PcD que tienen dificultades visuales (39,9\%) y auditivas (35\%) reportan las tasas de participación más altas. ${ }^{33}$

La primera complicación hallada al hablar de PcD mental o psicosocial en situación de calle es que no existen censos que ofrezcan una aproximación a la cantidad de personas que se encuentran en tales condiciones; este grupo vulnerable continúa en la oscuridad, marginado por el Estado. Es imperativo que este conozca y registre

33 Secretaría de Relaciones Exteriores, "Informes periódicos segundo y tercero combinados que México debía presentar en 2018 en virtud del artículo 35 de la Convención", http://docstore.ohchr.org/SelfServices/ FilesHandler.ashx?enc=6QkGld\%2fPPRiCAqhKb7yhskE4iNFvKWCCGr4TiTUdbhokZUaEzPwsH0KqV Tvlzw9bNWXQJVdJSj80Uv4Z\%2bZEgqoG8S\%2fvl\%2fmcFDGvJOPZmj3GsRfo4dHu0OysmnkzdQNbC (acceso octubre 26, 2018). 
la prevalencia de este fenómeno, con el fin de implementar estrategias oportunas para la solución de la problemática. Sin duda alguna, contar con estas cifras no implica superar el fenómeno, pero el hecho de no tenerlas denota nulo interés por parte del Estado.

Las PcD mental o psicosocial en situación de calle conforman uno de los grupos vulnerables más desprotegidos del país. La propia característica de su padecimiento les impide tener la capacidad intelectual para acudir por sus propios medios a una clínica de salud pública para recibir atención médica y mucho menos para hacer valer sus derechos. Ante esta situación, se encuentran en un total abandono por el Estado, por lo que es necesario que se reconozcan como un colectivo con derechos humanos que requieren protección del Estado. ${ }^{34}$

El Estado se escuda en cuestiones económicas para justificar la falta de políticas públicas o estrategias para dar una solución integral a esta problemática, pero ningún pretexto valida la violación de los derechos humanos de persona alguna, mucho menos de PcD mental o psicosocial en situación de calle, dadas sus circunstancias y que no cuentan con el mínimo vital.

El derecho al mínimo vital, según nuestra Suprema Corte de Justicia, tiene fundamento en los Artículos 31 y 123 de la CPEUM y es un mínimo de subsistencia con el que todo ser humano debe contar. Al Estado le corresponde tomar todas las medidas a su alcance, acordes con el Estado social de derecho, para proteger la dignidad de todas aquellas personas en una situación de desgracia como la aludida.

La protección de la salud de estos grupos en situación de vulnerabilidad debe ser referida en un apartado específico, porque en el panorama general que ha mostrado el Sistema Nacional de Salud no se aprecian las circunstancias particulares que demandan atención especializada. ${ }^{35} \mathrm{Al}$ hablar del derecho a la igualdad respecto al derecho a la salud, debemos entender que no es literal, es decir, se debe de tratar igual a los iguales y desigual a los desiguales, para lograr un sistema de protección

34 Carlos Parra y Beatriz Londoño, "La protección de la población con discapacidad en la jurisprudencia de la Corte Constitucional" en Derechos humanos y discapacidad, ed. Carlos Parra (Bogotá: Universidad del Rosario, 2004), 198.

35 Horacio García Romero y Fernando Cano Valle, El derecho a la protección de la salud de todos los mexicanos (Ciudad de México: Comisión Nacional de Derechos Humanos, 1996), 32. 
de la salud que brinde a las personas oportunidades iguales para disfrutar del mayor bienestar posible. ${ }^{36}$

Gutiérrez Calles hace una reflexión muy acertada respecto a las PcD mental o psicosocial, cuando afirma que, por su magnitud y su cualificación, la discapacidad mental plantea problemas de orden muy diferente que las discapacidades funcionales o físicas, a las que también debe proteger el derecho. Donde más se revela y más se interpela el núcleo duro de los derechos humanos es en situación de la discapacidad mental, porque sitúa a la persona en la frontera de la existencia. Frente a este hecho problemático, una ciencia normativa como el derecho tiene que interrogarse cómo y qué puede y tiene que hacer para el reconocimiento real de los derechos humanos (garantías, medios operativos, instrumentos) del discapacitado mental, con el objeto de devolverle su condición humana y de sacarlo de su situación de exclusión social. ${ }^{37}$

\section{Conclusiones}

Las personas con PcD mental o psicosocial en situación de calle deben ser concebidas como titulares de derechos a proteger por parte del Estado. Estas personas, como cualquier otra, gozan de los derechos inherentes a su persona, como el derecho a la salud, que se encuentra tutelado en diversos instrumentos internacionales, así como en nuestra CPEUM.

Sin embargo, es preocupante ver que aun el sistema internacional de los derechos humanos no les ha dado la importancia debida, en virtud de que no existe algún tratado vinculante ex profeso para su protección. Por la gran complejidad de su condición, requieren ser consideradas como uno de los grupos vulnerables más desprotegidos y por eso, crear las condiciones y estrategias necesarias para la protección de sus derechos.

Si bien no existe un instrumento internacional ex profeso para la protección de los derechos humanos de las PcD mental o psicosocial, todos recogen y protegen

36 Organización de las Naciones Unidas [ONU], Recopilación de las observaciones generales y recomendaciones generales adoptadas por órganos creados en virtud de tratados de derechos humanos (Nueva York: Instrumentos internacionales de derechos humanos, 2008), 96.

37 José Luis Gutiérrez Calles, La guarda del enfermo mental: deberes y responsabilidades (Madrid: Dykinson, 2004), 56 . 
los derechos de este grupo como los de cualquier otro, por lo que la obligación positiva del Estado se encuentra vigente.

Las PcD mental o psicosocial en situación de calle no deben de ser vistas como agentes que, por medio de políticas públicas "caritativas" o de "buena voluntad", serán rescatados de su condición. La implementación de estrategias para la protección de la salud de estas personas es una obligación constitucional del Estado. No se trata de que este, por medio de la caridad, las rescate de la ignominia; no es un acto de caridad, sino de resarcimiento del goce y ejercicio de sus derechos humanos. Es un acto de justicia.

Es sumamente necesario que las PcD mental o psicosocial en situación de calle tengan acceso urgente a los servicios de salud elementales y especializados, así como también a programas eficientes de protección y resguardo, ya que su condición se ve agravada día a día.

\section{Referencias}

Barahona-Riera, Rocío. "Derechos económicos, sociales y culturales: exigibilidad —niveles mínimos de cumplimiento- e indicadores". Ponencia presentada en la Reunión de expertos sobre población, desigualdades y derechos humanos. Santiago de Chile, 26-27 octubre, 2006.

Castilla Juárez, Karlos. "Un nuevo panorama constitucional para el derecho internacional de los derechos humanos en México". Estudios constitucionales 9, núm. 2 (junio-diciembre 2011): 123-164. https://scielo.conicyt.cl/pdf/estconst/v9n2/art04.pdf (acceso octubre 26, 2018).

Centro de Información de la Organización de Naciones Unidas. "Día internacional de las personas con discapacidad". http://cinu.mx/eventos/observancia/dia-internacionalde-las-perso-4/ (acceso octubre 26, 2018).

Comisión Interamericana de Derechos Humanos. Principios para la protección de los enfermos mentales y el mejoramiento de la atención de la salud mental. Washington, 17 de diciembre de 1991.

Estados Unidos de México, Cámara de Diputados. Ley general de salud. Ciudad de México: Diario Oficial de la Federación, 7 de febrero de 1984.

Estados Unidos de México, Congreso del estado. Ley de salud pública para el estado de Baja California. Mexicali: Periódico Oficial núm. 49, 9 de noviembre de 2001. 
Estados Unidos Mexicanos, Presidencia de la República. Decreto por el que se reforma y adicionan diversas disposiciones de la Constitución Politica de los Estados Unidos Mexicanos. Ciudad de México: Diario Oficial de la Federación, 20 de junio de 1983.

Estados Unidos Mexicanos. Constitución Política. Ciudad de México: Sista, 2017 [1917].

Ferreirós Marcos, Carlos-Eloy. Salud mental y derechos humanos: la cuestión del tratamiento ambulatorio involuntario. Madrid: Comité español de representantes de personas con discapacidad, 2007.

Ferrer Mac-Gregor, Eduardo. "El control difuso de convencionalidad en el Estado constitucional" en Formación y perspectivas del Estado en México, editado por Héctor Fix-Zamudio y Diego Valadés, 151-188. Ciudad de México: Universidad Nacional Autónoma de México, 2010.

García Romero, Horacio y Fernando Cano Valle. El derecho a la protección de la salud de todos los mexicanos. Ciudad de México: Comisión Nacional de Derechos Humanos, 1996.

Gutiérrez Calles, José Luis. La guarda del enfermo mental: deberes y responsabilidades. Madrid: Dykinson, 2004.

Organización de Naciones Unidas [ONU]. De la exclusión a la igualdad. Hacia el pleno ejercicio de los derechos de las personas con discapacidad. Ginebra: SRO-Kundig, 2007.

Organización de Naciones Unidas [ONU]. Declaración universal de los derechos humanos. Nueva York, 10 de diciembre de 1948.

Organización de Naciones Unidas [ONU]. Observaciones finales sobre el Informe inicial de México. Nueva York: Comité sobre los derechos de las personas con discapacidad, 2014. Organización de Naciones Unidas [ONU]. Pacto internacional de derechos económicos, sociales y culturales. Nueva York, 16 de diciembre de 1966.

Organización de Naciones Unidas [ONU]. Protocolo facultativo de la Convención sobre los derechos de las personas con discapacidad. Nueva York, 13 de diciembre de 2006.

Organización de Naciones Unidas [ONU]. Recopilación de las observaciones generales y recomendaciones generales adoptadas por órganos creados en virtud de tratados de derechos humanos. Nueva York: Instrumentos internacionales de derechos humanos, 2008.

Parra, Carlos y Beatriz Londoño. "La protección de la población con discapacidad en la jurisprudencia de la Corte Constitucional" en Derechos humanos y discapacidad, editado por Carlos Parra, 198-274. Bogotá: Universidad del Rosario, 2004.

Saldaña, Javier. Derechos del enfermo mental. Ciudad de México: Instituto Politécnico Nacional, 2000.

Secretaría de Relaciones Exteriores. "Informes periódicos segundo y tercero combinados que México debía presentar en 2018 en virtud del artículo 35 de la Convención”. http://docstore.ohchr.org/SelfServices/FilesHandler.ashx?enc=6QkG1d\%2fPPRiCA qhKb7yhskE4iNFvKWCCGr4TiTUdbhokZUaEzPwsH0KqVTvlzw9bNWXQJVdJS 
j80Uv4Z\%2bZEgqoG8S\%2fvl\%2fmcFDGvJ0PZmj3GsRfo4dHu0OysmnkzdQNbC (acceso octubre 26, 2018).

Secretaría de Relaciones Exteriores. Informe inicial de México sobre el cumplimiento de la Convención sobre los derechos de las personas con discapacidad. Ciudad de México: Secretaría de Relaciones Exteriores, Dirección general de derechos humanos y democracia, 2011. 